\title{
Healthcare Accessibility and Socio-economic Deprivation: A Case Study in Quito, Ecuador
}

\author{
Pablo Cabrera Barona and Thomas Blaschke \\ Department of Geoinformatics - Z_GIS, University of Salzburg, Austria · \\ pablo.cabrera-barona@stud.sbg.ac.at
}

Full paper double blind review

\begin{abstract}
Comparing different healthcare accessibility measures and relating them to socio-economic information is a useful means for identifying health inequalities. In this study, a socioeconomic deprivation index is initially calculated. Then, three measures of health services accessibility are calculated: distance to the nearest health center, a two-step floating catchment area measure, and a modified gravity-based accessibility measure. Finally, the three accessibility measures are compared to the socio-economic deprivation index. The study is applied in a study case in Quito, Ecuador. Results show differences between the different healthcare accessibility measures, and that areas with more socio-economic deprivation can be considered as areas that have limited access to health services.
\end{abstract}

\section{Introduction}

Geographical studies about healthcare have been very useful in identifying spatial health inequalities (BARNETT et al. 2002, OLIVER \& HAYES 2007). Measuring socio-economic deprivation is a proven strategy in supporting the identification of these health inequalities (SCHUURMAN et al. 2007, LALloué et al. 2013), and there is some evidence that socioeconomically deprived areas can have poor access to community resources (PEARCE et al. 2007). Accessibility is a multidimensional concept where the location of services influences the configuration of under-served areas (RADKE \& MU 2000), and, in the case of health services, it can be concluded that distance is a determinant for the people when choosing to utilize these kinds of services (VERTER \& LAPIERRE 2002). There are several methods to calculate healthcare accessibility, such as gravity model-based methods (KWAN 1998, Guagliardo 2004, CROOKS \& SCHUURMAN 2012), and the methods that use floating catchment areas (RADKE \& MU 2000, WANG \& LUO 2005, LUO \& WHIPPO 2012). However, it is important to compare and evaluate them in order to obtain an optimization of the services that could support a reduction of disparities in health outcomes (WANG 2012). Comparing accessibility methods and relating them to socio-economic deprivation can support a better understanding of the effectiveness of health accessibility measures, and can also support the identification of under-served areas in more deprived zones. This study compares different healthcare accessibility measures to socio-economic deprivation and uses the results of this relation in order to determine whether poorer people have poorer access to health services.

GI_Forum - Journal for Geographic Information Science, 1-2015.

(C) Herbert Wichmann Verlag, VDE VERLAG GMBH, Berlin/Offenbach. ISBN 978-3-87907-558-4.

(C) ÖAW Verlag, Wien. ISSN 2308-1708, doi:10.1553/giscience2015s484. 


\section{Input Data and Data Processing}

This study uses information obtained from the 2010 Ecuadorian Population and Housing Census, provided by the Ecuadorian Institute of Census and Statistics. Data from public health centers, provided by the Ministry of Health of Ecuador, is also used.

The study area is the urban parish of "El Condado", located in the north-west of the Metropolitan District of Quito (MDQ), Ecuador. This area could practically be considered a small city, with a population of 85844 inhabitants. The parish includes different socio-economic neighborhoods, from lower-income neighborhoods (such as Pisulí and Roldós), to wealthy neighborhoods (such as El Condado).

The study area has 229 census blocks. The following indicators were extracted from the 2010 Census: the percentage of houses without a connection to the sewerage system, the percentage of houses without a connection to the electric system, the percentage of houses without a connection to the drinking water supply system, and the percentage of houses without a garbage collection service. These indicators were geo-coded and were then linked to the 229 census blocks. Based on the public health centers data, eight geo-referenced health centers were used for this study. Out of these eight, six centers are public health services assigned to offer public healthcare in the parish, and an additional two centers were considered because they are located less than 300 meters from the parish boundary.

\section{Methods}

\subsection{Calculation of Socio-economic Deprivation Index}

A socio-economic deprivation index was calculated following a rights-based perspective (MIDEROS 2012). To calculate the index, the following four indicators were used: the percentage of houses without a connection to the sewerage system, the percentage of houses without a connection to the electric system, the percentage of houses without a connection to the drinking water supply system, and the percentage of houses without a garbage collection service. Multi-collinearity was evaluated using Variances Inflation Factors (VIF) (OECD 2008). All the VIF obtained were lower than 5, thus all the indicators could be used to construct the deprivation index. The deprivation index was calculated by applying a simple linear combination:

$$
\sum_{i=1}^{i=4} I_{i}
$$

where $I_{i}$ represents each indicator used. The final deprivation index was obtained by applying a min-max normalization (MALCZEWSKI 1999):

$$
v_{i}=\frac{v_{i}-v_{\min }}{v_{\max }-v_{\min }}
$$


where $v_{i}$ is the non-normalized value of the index, $v_{\min }$ is the lower value of the index and $v_{\max }$ is highest value of the index. The final result is an index with values between 0 and 1 , where values closer to 1 represent higher socio-economic deprivation.

\subsection{Calculation of Health Accessibility Measures}

Considering the spatial location of the census blocks and the health services, three measures of access to health services were calculated. First, the centroids of each census block were generated.

The first measure of access to the health centers was the distance from each census block $i$ centroid, to the nearest health center $\left(D_{i}\right) . D_{i}$ represents the first health accessibility measure.

For the second measure of access to the health centers, the two-step floating catchment area (2SFCA) measure was calculated (RADKE \& MU 2000, WANG \& LUO 2005). This method works with a distance threshold from each population location to each health center. For this study, a distance threshold of 1 kilometer was defined because this distance is considered a walkable distance to the health centers in the study area, and because the health centers are located relatively close to each other, including two health centers that are located a few meters outside the parish boundary. The chosen distance threshold also avoids an overestimation of accessibility, since the study area is not a large rural zone (BELL et al. 2013).

To calculate the 2SFCA, all the population centers (represented by the census block centroids) that were located inside the 1 kilometer distance threshold were identified for each health center: a 1 kilometer buffer was generated for each health center, and all the population centers inside a health center buffer were considered to represent the population using the specific health center. The population information of each health center was extracted from the 2010 Census.

The first step of the 2SFCA is to calculate the provider to population ratio (WANG \& LUO 2005):

$$
R_{j}=\frac{S_{j}}{\sum_{k \in\left\{d_{k j} \leq d_{0}\right\}} P_{k}}
$$

where $S_{j}$ is a number of healthcare staff at the health center $j$. All health centers have an $S_{j}$ value of 4 because they are primary healthcare centers of type A. The Ecuadorian Health System established that a type A health center should have 4 healthcare staff members: one physician, one specialist of primary healthcare, one dentist, and one extra healthcare professional depending on the needs of the local population. $\mathrm{P}_{\mathrm{k}}$ represents the population of a census block $k, \mathrm{~d}_{0}$ is the distance threshold of 1 kilometer.

The second step of the 2SFCA is to calculate the accessibility measure:

$$
A_{i}=\sum_{j \in\left\{d_{j} \leq d_{0}\right\}} R_{j}
$$

where $R_{j}$ represents all the ratios of the health centers that are located inside a 1 kilometer ratio of each population center. $A_{i}$ represents the second health accessibility measure used in this study. 
To calculate the third measure of access to the health centers, a modified gravity-based accessibility measure was adapted for the study area. The following equation was used to calculate this measure (GUAGLIARDO 2004):

$$
G_{i}=\sum_{j} \frac{S_{j}}{d_{k j}^{\beta}}
$$

where $S_{j}$ is the number of healthcare staff, and $\mathrm{d}_{\mathrm{kj}}$ is the equivalent to the first accessibility indicator used in this study $\left(D_{i}\right)$. For this study, $\mathrm{d}_{\mathrm{kj}}$ with the $\beta$ exponent is defined as the "travel impedance factor" (GUAGLIARDO 2004) of all population centers located inside the 1-kilometer range of influence of each health center. $\beta$ is the friction-of-distance coefficient, and depending on physician-patient interaction data (LUO \& QI 2009, LUO \& WHIPPO 2012) its value varies from place to place and over time (WANG \& LUO 2005). For this study, a value of 1 was chosen for $\beta$ under the assumption that the distance to a health center and the number of people choosing to make use of the health center is inversely proportional. This means that when the distance to a health center is doubled, the willingness of people to visit this health center reduces by half (SCHUURMAN et al. 2010). $G_{i}$ represents the third health accessibility measure used in this study.

In order to gain a better understanding of the accessibility variations of the measures $A_{i}$ and $G_{i}$, all their values were normalized by applying min-max normalization. This normalization does not have any impact on the evaluation of the relationships between these measures and the socio-economic deprivation index.

\subsection{Relationship Between Socio-economic Deprivation Index and Accessibility Measures}

To evaluate the relationships between the socio-economic deprivation index and accessibility measures, Pearson correlation coefficients were calculated.

\section{Results and Discussion}

Our socio-economic deprivation index varies from 0 to 1 , and most of the census blocks have deprivation values lower than 0.3 . Figure 1 shows that most of the households in the study area have good socio-economic conditions (low deprivation). Only some peripheral census blocks located in the west of the El Condado parish lack some of the services mentioned above. 


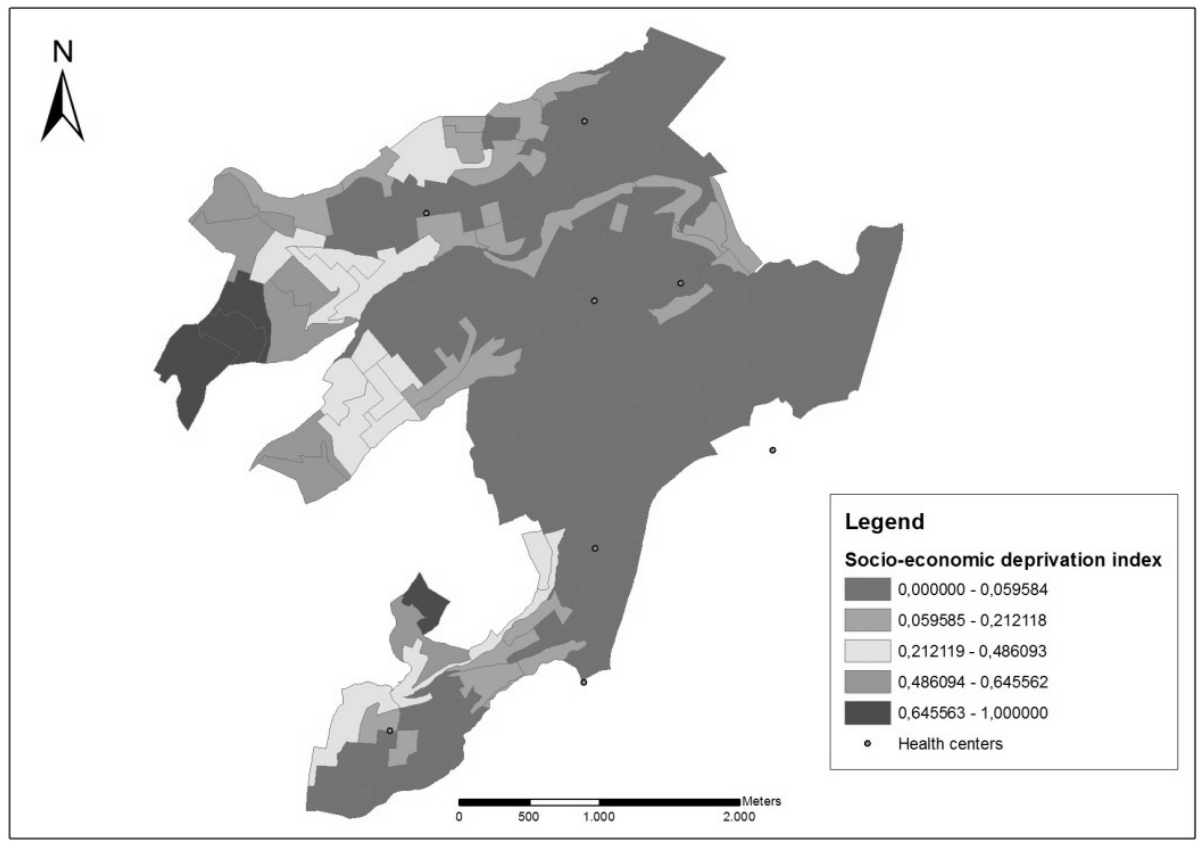

Fig. 1: Socio-economic deprivation index

Figure 2 shows three maps of the different health accessibility measures in the study area. The red color shows the least accessibility, followed by the orange, yellow, and finally the green tones, which represent the areas with the best healthcare accessibility. The map of the measure of distance to the nearest health center $\left(D_{i}\right)$ (figure 2a) shows a spatial correlation between the health centers' location and the census blocks with more accessibility. In the second map (figure $2 \mathrm{~b}$ ), the two-step floating catchment area measure $\left(A_{i}\right)$, shows overestimations and underestimations in accessibility values. Some areas that include health centers appear to have lower accessibility scores compared to other areas that do not contain health services. Even when there are some overestimations and underestimations, due to the fact that this measure considers not only the provider to population ratio but the health centers that are located inside a search area from population centers, it could be concluded that the two-step floating catchment area methodology is useful to identifying in which population areas there is less "competition" for healthcare access. This means that green areas with better levels of accessibility have more healthcare resources available than the red ones. Finally, the third measure, the modified gravity-based accessibility $\left(G_{i}\right)$, shows characteristics from both measures mentioned before: higher values of accessibility are present in areas closer to health centers, and it can also be observed that some underestimations occur in areas that are very close to a health service, even though they do not include one within their borders (figure 2c). 


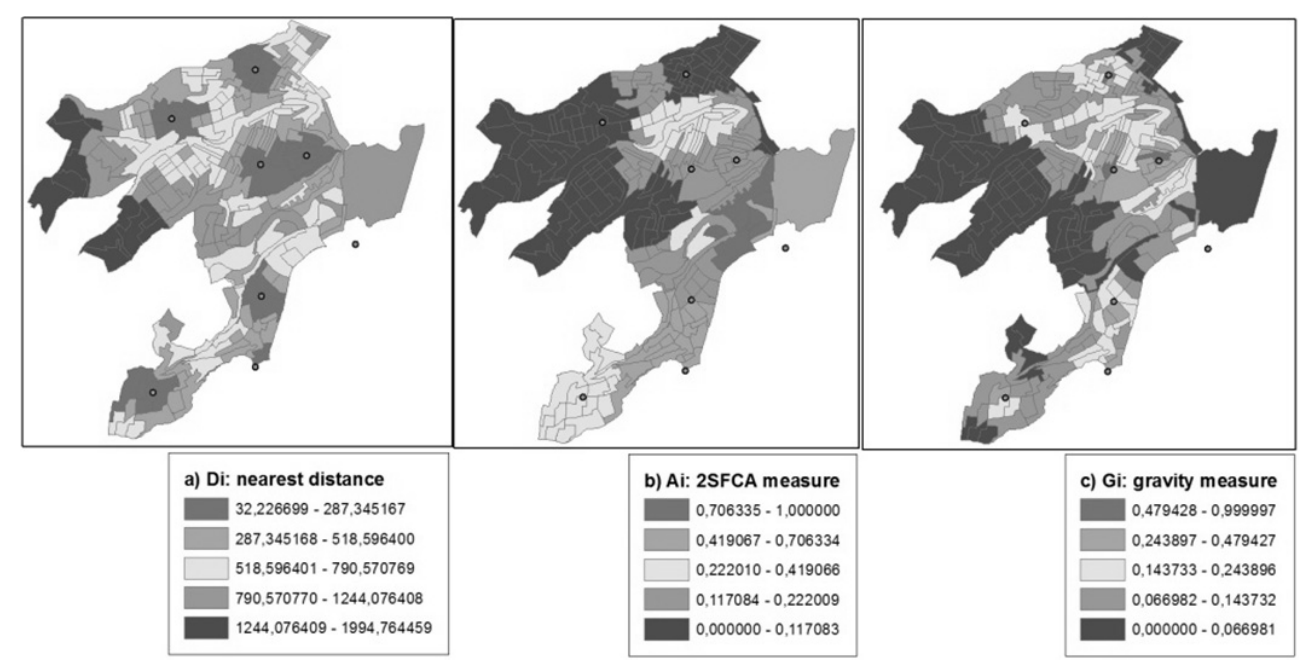

Fig. 2: Health accessibility measures: a) distance to the nearest health center measure $\left(D_{i}\right)$; b) two-step floating catchment area measure $\left(A_{i}\right)$; c) modified gravity-based accessibility measure $\left(G_{i}\right)$

The distance to the nearest health center measure $\left(D_{i}\right)$ shows a strong linear correlation with the socio-economic deprivation index (0.62). The two-step floating catchment area measure $\left(A_{i}\right)$ and the modified gravity-based accessibility measure $\left(G_{i}\right)$ correlate negatively to socioeconomic deprivation: -0.21 and -0.29 , respectively.

Figure 3 shows the scatterplots of the correlations between the socio-economic deprivation index and the three health accessibility measures. Pearson coefficients show a clear relationship: a higher level of socio-economic deprivation correlates to a lower accessibility to health services. However, the scatterplots do not show a perfect linear correlation between the variables in question.

The first scatterplot refers to the distance to the nearest health center measure $\left(D_{i}\right)$ and it shows that areas with values of deprivation equal or close to zero are areas located less than 1 kilometer from a health center. The second scatterplot, related to the two-step floating catchment area measure $\left(A_{i}\right)$, shows that census blocks with very low socio-economic deprivation have different accessibility values: from 0 to 1 . The third scatterplot refers to the gravity-based accessibility measure $\left(G_{i}\right)$, and it shows a majority of census blocks that have low levels of accessibility to health centers and very low values of socio-economic deprivation.

Calculating different accessibility measures leads to different results depending on the concepts and variables used (GUAGLIARDO 2004, APPARICIO et al. 2008). Cartesian measures, such as the distance to the nearest health center $\left(D_{i}\right)$ calculated in this study, have been shown to be very efficient when evaluating healthcare access in urban areas (APPARICIO et al. 2008). $D_{i}$ also proved to be a very useful accessibility measure when comparing healthcare access to socio-economic deprivation. Both measures could support decision makers in taking action in urban zones with higher levels of deprivation and less access to healthcare services. A critical zone identified in our study is a zone located in the extreme north-west of the study area. This zone shows high levels of deprivation and low levels of accessibility 
a

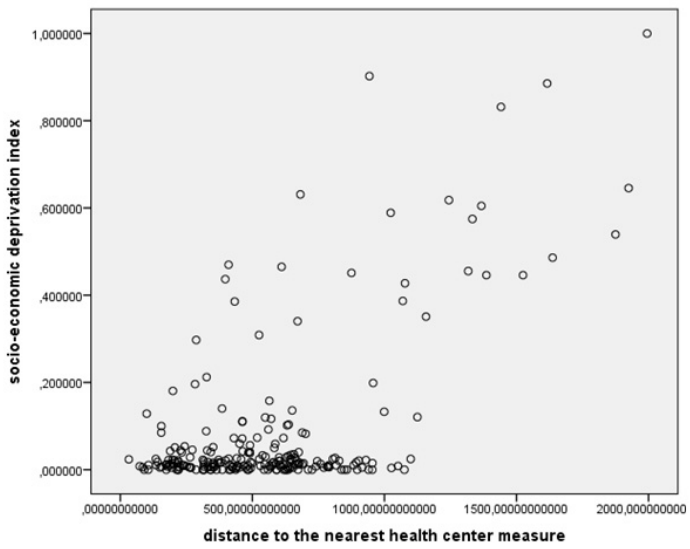

b
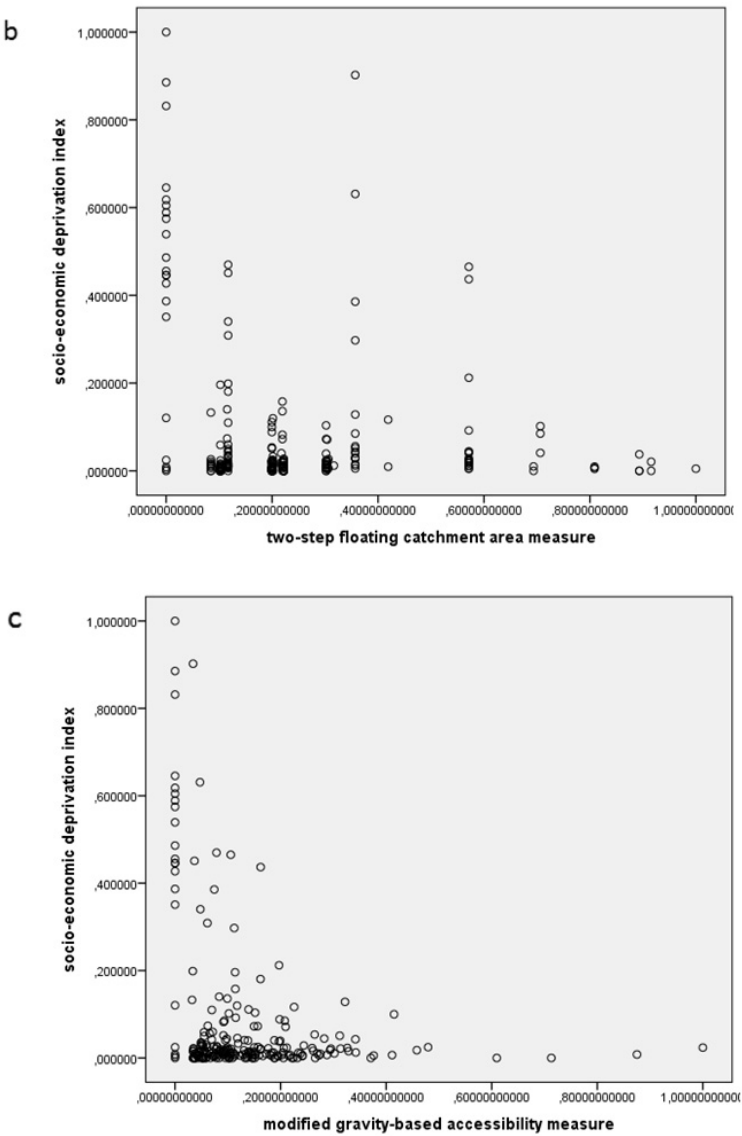

Fig. 3: Scatterplots of the correlations between the socio-economic deprivation index and the three health accessibility measures: a) distance to the nearest health center measure $\left(D_{i}\right)$; b) two-step floating catchment area measure $\left(A_{i}\right)$, c) modified gravity-based accessibility measure $\left(G_{i}\right)$ 
to health centers in the three measures used. Major and urgent social investment should be applied in this critical zone. Social investment should also be considered for the areas surrounded this critical zone.

The two-step floating catchment area measure $\left(A_{i}\right)$ and the modified gravity-based accessibility measure $\left(G_{i}\right)$ are measures based on ratios and buffers, and an oversimplifycation due to different accessibility degrees that are present in real life could occur in these kinds of approaches (BELL et al. 2013). However, results of $A_{i}$ and $G_{i}$ could offer a general view of areas that are less affected by a lack of healthcare access. For our study, some zones located in the center and south of the study area have higher levels of accessibility to health centers. These results are confirmed by our first and more effective accessibility measure, $D_{i}$.

\section{Conclusion and Outlook}

This work found that more deprived people generally have poorer access to health services in the study area. The distance to the nearest health center measure $\left(D_{i}\right)$ was shown to be an effective measure of health accessibility for the case study, and both the two-step floating catchment area measure $\left(A_{i}\right)$ and the modified gravity-based accessibility measure $\left(G_{i}\right)$ underestimate and overestimate accessibility in some census blocks. This is possibly due to an oversimplification of different accessibility degrees that are present in real life. Another reason could be that our study area is not as large an area as some rural zones, and that it includes several health centers: high potential demand of healthcare and an acceptable number of health centers in a not so large area could have led to misinterpretations in some census blocks when using the two-step floating catchment area and the modified gravitybased accessibility measures. This study is a contribution to the health accessibility and socio-economic deprivation literature, and could be a guide for researchers that are interested in comparing measures of healthcare accessibility. Indeed, future work related to the topics examined in this study should focus on 1) evaluating the scale effects of different health accessibility and deprivation measures; 2) searching for a maximization of healthcare accessibility for more deprived zones; 3) considering more operational/methodological aspects, such as considering distance decay functions that include specific parameters for a study area; and 4) evaluating the influence of access to healthcare and socio-economic deprivation on specific health problems or illnesses. Finally, this study could also be considered as a current status report of socio-economic deprivation and health accessibility in the study area, with results that can support decision making of health planners and authorities in the Metropolitan District of Quito.

\section{References}

Apparicio, P., Abdelmajid, M., Riva, M. \& Shearmur, R. (2008), Comparing alternative approaches to measuring the geographical accessibility of urban health services: Distance types and aggregation-error issues. International Journal of Health Geographics, 7 (7).

Barnett, P., Roderick, P., MARtin, D., Diamond, I. \& Wrigley, H. (2002), Interrelations between three proxies of health care need at the small area level: an urban/rural comparison. Journal of Epidemiological Community Health, 56, 754-761. 
Bell, S., Wilson, K., Bissonnette, L. \& Shah, T. (2013), Access to Primary Health Care: Does Neighborhood of Residence Matter? Annals of the Association of American Geographers, 103 (1), 85-105.

CROOKS, V. \& SCHUURMAN, N. (2012), Interpreting the results of a modified gravity model: examining access to primary health care physicians in five Canadian provinces and territories. Health Services Research, 12 (230).

GuAgLiARDO, M. (2004), Spatial accessibility of primary care: concepts, methods and challenges. International Journal of Health Geographics, 3 (3).

KwAN, M. (1998), Space-Time and Integral Measures of Individual Accessibility: a Comparative Analysis Using a Point-based Framework, 30 (3), 191-216.

Lalloué, B., Monnez, J., Padilla, C., Kihal, W., Le Meur, N., Zmirou-Navier, D. \& DEGUEN, S. (2013), A statistical procedure to create a neighbourhood socioeconomic index for health inequalities analysis. International Journal of Equity in Health, 12 (21).

LUO, W. \& QI, Y. (2009), An enhanced two-step floating catchment area (E2SFCA) method for measuring spatial accessibility to primary care physicians. Health \& Place, 15, 11001107.

LUO, W. \& WHIPPO, T. (2012), Variable catchment sizes for the two-step floating catchment area (2SFCA) method. Health \& Place, 18, 789-795.

MALCZEWSKI, J. (1999), GIS and multicriteria decision analysis. Wiley, New York, 392 p.

Mideros, A. (2012), Ecuador: defining and measuring multidimensional poverty, 20062010. CEPAL Review, 108, 49-67.

OECD (2008), Handbook on constructing composite indicators: methodology and user guide. $160 \mathrm{p}$.

Oliver, L. N. \& HAYeS, M. V. (2007) Does Choice of Spatial Unit Matter for Estimating Small-area Disparities in Health and Place Effects in the Vancouver Census Metropolitan Area? Canadian Journal of Public Health, 98 (1), S27-S34.

Pearce, J., Witten, K., Hiscock, R. \& Blakely, T. (2007), Are socially disadvantaged neighbourhoods deprived of health-related community resources? International Journal of Epidemiology, 36, 348-355.

RADKE, A. \& MU, L. (2000), Spatial decomposition, modelling and mapping service regions to predict access to social programs. Geographic Information Sciences, 6, 105-112.

Schuurman, N., Bell, N., DunN, J. R., \& Oliver, L. (2007), Deprivation Indices, Population Health and Geography: An Evaluation of the Spatial Effectiveness of Indices at Multiple Scales. Journal of Urban Health: Bulletin of the New York Academy of Medicine, 84 (4), 591-603.

SChUURMAN, N., BÉRUBÉ, M. \& CROOKS, V. A. (2010), Measuring potential spatial access to primary health care physicians using a modified gravity model. The Canadian Geographer, 54 (1), 29-45.

Verter, V. \& LAPIERRE, S. (2002), Location of Preventive Health Care Facilities. Annals of Operations Research, 110, 123-132.

WANG, F. (2012), Measurement, Optimization, and Impact of Health Care Accessibility. Annals of the Association of American Geographers, 102 (5), 1104-1112.

WANG, F. \& LUO, W. (2005), Assessing spatial and nonspatial factors for healthcare access: towards an integrated approach to defining health professional shortage areas. Health \& Place, 11, 131-146. 